\title{
Targeting physiotherapy resources - evaluation of the southampton physiotherapy post-operative screening tool (SPPOST)
}

\author{
J Weblin*, DJ McWilliams \\ From ESICM LIVES 2015 \\ Berlin, Germany. 3-7 October 2015
}

\begin{abstract}
Introduction
The benefit and necessity for prophylactic physiotherapy post operatively remains unclear [1]. Combined with an increased demand on resources, scores to identify those patients who would most benefit are being increasingly used. The SPPOST is a tool to identify patients who are at high risk of developing post-operative pulmonary complications (PPC's). A previous trial of its implementation demonstrated physiotherapy to be safely withdrawn from $46 \%$ of post-operative patients with minimal risk [2].
\end{abstract}

\section{Objectives}

To assess the feasibility of the SPPOST as an effective tool in prioritising patients for post-operative physiotherapy treatment within a large UK based specialist surgery service.

\section{Methods}

All patients undergoing abdominal surgical procedures between $29^{\text {th }}$ August and $5^{\text {th }}$ October 2013 were included in the analysis. Patients were excluded if they had their operation at a weekend. SPPOST scores were calculated on the first post-operative day, with a score $\geq 10$ identifying a patient as high risk and requiring physiotherapy assessment and intervention. Physiotherapists were blinded to the threshold score and treatment was delivered based on individual clinical reasoning. Patients were assessed daily for the development of a PPC using the Brooks-Brunn criteria. Data was analysed using the Chi squared test.

\section{Results}

Ninety three patients were included in the analysis, with 54 (58\%) deemed high risk. More PPC's were identified

UHB NHS Foundation Trust, Birmingham, United Kingdom in the high risk patients, although this was not significant $(9,17 \%$ vs $3,8 \% ; p=0.20)$.A subsequent analysis identified 16 low risk patients having received physiotherapy, whilst 14 high risk patients were not assessed by a physiotherapist. It was noted junior members of staff were more likely to screen out high risk patients.

\section{Conclusions}

A threshold score of 10 appeared to be sensitive for identifying patients at higher risk of developing PPC's. Although not statistically significant, it is acknowledged as a pilot project that the study may not have been appropriately powered. A sub analysis of the data suggested the SPPOST could provide a structured and robust approach to identifying post-operative patients who would most benefit from physiotherapy, particularly for junior members of staff. It is acknowledged that a number of patients in the low risk category still received physiotherapy, which may have reduced the incidence of PPC's observed. Appropriately powered trials using the SPPOST as a screening tool for physiotherapy input are therefore needed to confirm its effectiveness.

Published: 1 October 2015

\section{References}

1. Pasquina P, Tramer MR, Granier JM, Walder B: Respiratory physiotherapy to prevent pulmonary complications after abdominal surgery. Chest 2006, 130(6):1887-1889.

2. Ostler CM, van Willigen ZE, Gibson D, Devlin R, Bruton A: Prioritising physiotherapy services The development andimplementation of a postoperativescreening tool. ACPRC Journal 2008, 40:23-30.

doi:10.1186/2197-425X-3-S1-A549

Cite this article as: Weblin and McWilliams: Targeting physiotherapy resources - evaluation of the southampton physiotherapy postoperative screening tool (SPPOST). Intensive Care Medicine Experimental 2015 3(Suppl 1):A549. 